\title{
Reflets
}

Revue d'intervention sociale et communautaire

\section{SHEEHY, Elizabeth A (2014). Defending battered women on trial : Lessons from the transcripts, UBC Press, 469 p.}

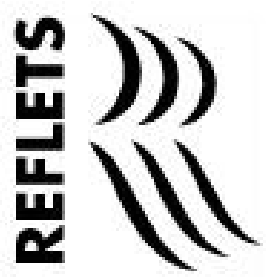

\section{Vanessa Couturier}

Volume 21, numéro 1, printemps 2015

URI : https://id.erudit.org/iderudit/1032559ar

DOI : https://doi.org/10.7202/1032559ar

Aller au sommaire du numéro

Éditeur(s)

Reflets, Revue d'intervention sociale et communautaire

ISSN

1203-4576 (imprimé)

1712-8498 (numérique)

Découvrir la revue

Citer ce compte rendu

Couturier, V. (2015). Compte rendu de [SHEEHY, Elizabeth A (2014). Defending

battered women on trial: Lessons from the transcripts, UBC Press, 469 p.]

Reflets, 21(1), 242-248. https://doi.org/10.7202/1032559ar d'utilisation que vous pouvez consulter en ligne.

https://apropos.erudit.org/fr/usagers/politique-dutilisation/ 


\section{Defending battered women on trial : Lessons from the transcripts}

SHEEHY, Elizabeth A (2014). UBC Press, 469 p.

par Vanessa Couturier

\section{Présentation}

Quel est le sort juridique des femmes qui ont recours à l'homicide de leur agresseur pour s'extraire de la violence conjugale? Defending battered women on trial nous propose une incursion dans l'univers du système judiciaire canadien où les femmes victimes de violences se retrouvent particulièrement désavantagées, à cause notamment de ses fondements historiques patriarcaux. Sheehy a analysé de façon approfondie les transcriptions de procès de 141 femmes accusées d'homicide de leurs conjoints entre 1990 et 2005. Elle expose le cas de onze d'entre elles, à savoir, un litige civil et dix procès criminels impliquant des femmes victimes de violence conjugale qui ont mis fin à la vie de leurs agresseurs. Divisé en sept chapitres encadrés d'une introduction et d'une conclusion, l'ouvrage explore par le biais d'analyses de cas les défis et dilemmes qu'elles ont vécus dans un système où les procès pour ces femmes s'avèrent plus hostiles que le seraient ceux des hommes. L'auteure propose de nombreuses réflexions quant à la place de la légitime défense dans les situations de violence conjugale et de sa place dans le cadre judiciaire ainsi qu'à l'argument de justification de l'acte homicidaire que l'on appelle le « syndrome de la femme battue ». 


\section{1 - Angelique Lyn Lavallee}

Defending battered women on trial s'ouvre sur le procès d'Angelique Lyn Lavallee ou l'affaire Lavallee. En 1990, ce procès a progressivement mené à une modification de la loi sur la légitime défense pour les femmes victimes de violence accusées d'homicide de leur agresseur, et ce, grâce entre autres au travail de la juge Bertha Wilson qui a entamé et alimenté chez les avocats canadiens une réflexion sur des thèmes et des concepts reliés à la violence conjugale.

À partir de la transcription du procès de Lavallee, Sheehy met en lumière le travail du conseiller juridique Greg Brodsky, qui s'est inspiré des travaux de féministes du battered women's movement pour défendre les femmes qui ont recours à l'homicide de leur agresseur. L'auteure introduit et critique également dans ce chapitre la question de l'utilisation de la théorie du syndrome de la femme battue afin d'" excuser " l'acte homicidaire, théorie qui ne tient pas compte de la problématique sociale dans laquelle s'inscrit l'homicide. L'utilisation flagrante du concept encourage d'ailleurs l'individualisation de la problématique de la violence conjugale en mettant l'accent sur la santé mentale des femmes et en ignorant les liens flagrants entre pouvoir, domination et violences des hommes à l'égard des femmes. Selon Sheehy, des alternatives à l'utilisation de la théorie du syndrome de la femme battue devraient être privilégiées, dont le " contrôle coercitif » et l'évidence du contexte social qui ne mènent pas à un étiquetage lié à un diagnostic pathologisant pour les femmes.

\section{2 - Bonnie Mooney}

Que peut-il arriver lorsque l'État ne parvient pas à assurer la sécurité de la femme victime de violence? Ce chapitre explore le cas de Bonnie Mooney qui a échappé à une tentative de fémicide de la part de son ex-conjoint, Roland Kruska. La nuit du 29 avril 1996, une arme à feu à la main, Kruska s'est présenté à la 
demeure de Mooney prenant la vie de l'amie de cette dernière, blessant gravement sa fille aînée et traumatisant sa fille cadette pour finalement se suicider. À quatre reprises déjà, Mooney avait tenté de dénoncer auprès des services policiers et du système judiciaire les mauvais traitements subis aux mains de son exconjoint; elle a intenté une poursuite civile contre le procureur général et le corps policier de la Colombie-Britannique pour avoir échoué à assurer sa protection.

Dans ce chapitre, Sheehy s'interroge sur les interventions policières et les options mises à la disposition des femmes pour se sortir de la violence. Elle y expose également le blâme continuel porté à l'égard des femmes au sein du système judiciaire. La poursuite de Mooney illustre d'ailleurs la dangerosité d'un discours favorisant le renvoi continuel à la faute de la femme qui ne quitte pas la relation violente.

C'est afin de déconstruire cette culture du blâme à l'égard des femmes que l'auteure aborde, à la suite de la juge Wilson dans l'affaire Lavallee, les facteurs sociaux pouvant empêcher les femmes de quitter une relation violente. Ces facteurs comprennent les inégalités économiques auxquelles elles doivent faire face, la présence d'enfants et la peur pour leur sécurité, et la crainte de représailles de la part du conjoint violent.

\section{3 - Kimberley Kondejewski}

Kimberley Kondejewski a été condamnée à perpétuité pour le meurtre de son conjoint violent, John Kondejewski. Sheehy dresse un bilan des nombreuses formes de violences subies par Kimberley et employées par son agresseur pour la contrôler, dont la menace qu'il allait s'en prendre aux enfants si elle ne commettait pas son propre suicide. L'auteure explore la question de l'accès à un procès juste et équitable pour les femmes victimes de violences qui, ayant tué pour assurer leur sécurité, sont condamnées à une sentence de prison à vie. 
S'appuyant sur la transcription du procès de Kimberley Kondejewski, Sheehy illustre les risques de l'utilisation du syndrome de la femme battue. Elle remet en question le concept étroit de la légitime défense là où un meurtre planifié effectué dans le but d'assurer la survie de la femme et de ses enfants n'est pas considéré par le système criminel canadien comme une forme de légitime défense.

\section{4 - Gladys Heavenfire and Doreen Sorenson}

Ce chapitre porte sur le racisme systémique que vivent les femmes autochtones du Canada et sur les liens entre le racisme, la violence conjugale et les interventions des acteurs au sein du système judiciaire. Survivantes de bon nombre de mesures assimilatrices et opprimantes effectuées par l'État depuis le colonialisme, les femmes autochtones sont aujourd'hui plus exposées à la violence conjugale et au fémicide que les non autochtones. À travers les transcriptions des procès de Gladys Heavenfire et de Doreen Sorenson, deux femmes autochtones ayant tué leurs conjoints non autochtones, Sheehy dénonce le racisme «silencieux » sous-jacent au système de justice. L'auteure remet en question l'utilisation du syndrome de la femme battue pour ces femmes qui présentent un vécu autre que celui des femmes non autochtones et elle dénonce les nombreux stéréotypes qui rendent l'argument de la légitime défense difficile à justifier dans leur cas.

\section{5 - Donelda Kay, Denise Robin Rain, and Jamie Gladue}

En lien avec le précédent, ce chapitre présente l'histoire de trois femmes autochtones victimes de violence qui ont tué leurs conjoints, mais autochtones eux aussi. À l'aide des transcriptions des procès de Donelda Kay, de Denise Robin Rain et de Jamie Gladue et en s'inspirant d'une étude réalisée par McGillivray 
et Comaskey concernant le rôle de la colonisation en lien avec l'expérience vécue par les femmes autochtones victimes de violence conjugale, Sheehy identifie des caractéristiques communes retrouvées dans les homicides commis par des femmes autochtones. Déclarées plus souvent coupables qu'innocentes par la cour, les femmes autochtones doivent faire face à des facteurs pouvant les forcer à plaider coupables, dont le racisme systémique et les barrières linguistiques et culturelles. C'est dans ce sens que l'auteure propose de contrer les stéréotypes à l'égard des femmes autochtones, qui sont davantage reconnues comme agresseuses plutôt que victimes, pour ainsi promouvoir un procès qui prend en compte des facteurs historiques et culturels spécifiques aux populations autochtones.

\section{6 - Lilian Getkate}

Le 8 décembre 1995, Lilian Getkate tue son conjoint violent, membre de la Gendarmerie royale du Canada, avec une arme à feu pendant qu'il sommeillait. À partir de la transcription du procès de Lilian Getkate, Sheehy s'interroge sur l'opportunité d'utiliser le concept de la légitime défense au sein du système judiciaire lorsque l'homicide s'est déroulé dans un contexte absent d'hostilité. Le cas de Lilian Getkate démontre que les formes de violences d'une ampleur considérable, telles que psychologiques et financières, ne sont pas considérées dans un plaidoyer s'appuyant du syndrome de la femme battue. C'est dans ce sens que l'auteure propose l'utilisation du concept de "contrôle coercitif" comme argument qui aurait dû être mis de l'avant à la cour pour le cas de Getkate. C'est aussi dans ce chapitre que Sheehy aborde la question des médias et de leur rôle dans l'attribution du blâme aux femmes victimes de violence conjugale et coupables d'homicide de leur agresseur. 


\section{7 - Margaret Ann Malott and Rita Graveline}

Ce chapitre traite des répercussions engendrées par l'oubli et la dissociation de la réalité pour les femmes victimes de violence ayant tué leur conjoint, compromettant ainsi l'invocation de la notion de la légitime défense à la cour. Afin d'oublier les conséquences associées aux diverses formes de violences qu'elles subissent, certaines femmes sombrent dans l'abus de substances, dont les drogues et l'alcool. L'abus de substances ainsi que les conséquences physiques provoquées par la violence de l'agresseur peuvent avoir de réelles répercussions sur la mémoire des femmes, ce qui peut jouer contre elles dans le cadre d'un procès.

À l'appui des transcriptions des procès de Margaret Ann Malott et de Rita Graveline et à partir des travaux de Stark et de Flitcraft, Sheehy critique le rôle des acteurs issus du milieu médical qui amplifient le piège social que vivent les femmes victimes de violence conjugale par la médicalisation de ses conséquences. L'auteure aborde également les abus psychologiques comme forme de destruction et conclut en se demandant si la légitime défense ne devrait pas être reconnue comme étant aussi psychologique.

\section{Réflexion}

Fruit d'une recherche rigoureuse regroupant minutieusement et stratégiquement des transcriptions de procès et des arguments issus de diverses disciplines scientifiques, Defending battered women on trial s'avère riche de par sa capacité à offrir aux lectrices et aux lecteurs l'occasion de plonger dans le vécu difficile de femmes qui ont recouru à l'homicide de leur agresseur pour s'extraire de la violence. L'ouvrage met également en lumière sur les enracinements idéologiques relevant du patriarcat que l'on retrouve ancré dans les interventions des divers acteurs représentant l'État. Les nombreuses comparaisons entre les transcriptions que propose l'auteure permettent également de comprendre la violence sous un angle structurel, et ce, malgré 
le constat que les interventions mises de l'avant par les institutions à l'égard de ces femmes amènent à l'individualisation et à la psychologisation d'une problématique que l'on retrouve partout au Canada. Dans sa conclusion, Sheehy propose des pistes de solutions concrètes afin de mettre un terme aux oppressions vécues par les femmes dans la société canadienne, en mettant l'accent sur des changements légaux, sociaux et économiques.

La lecture de Defending battered women on trial pourrait s'avérer intéressante, sinon essentielle, pour les personnes qui interviennent auprès des femmes victimes de violence conjugale, tant sein des services sociaux, hospitaliers ou policiers que juridiques. L'ouvrage est d'une pertinence particulière pour les intervenantes et les intervenants sociaux, puisqu'il les outille par le biais de la lecture de transcriptions de procès à reconnaitre les nombreux signes de violence faite par les hommes à l'égard des femmes. La reconnaissance de ces signes est d'une importance capitale puisque les intervenantes et les intervenants sociaux peuvent jouer un rôle clé pour guider et assurer la protection des femmes lorsqu'elles ne sont pas jugées crédibles par l'État. Enfin, écrit dans un vocabulaire clair et précis malgré la complexité et la lourdeur du sujet, l'ouvrage est également fortement recommandé à toute lectrice ou à tout lecteur voulant comprendre davantage la problématique de la violence conjugale.

L'ouvrage de Sheehy s'avère être une source importante et cruciale pour faire avancer la reconnaissance des femmes comme étant des êtres devant recevoir les mêmes droits que les hommes sur le plan du système judiciaire et est un outil d'éducation puissant pour dénoncer et faire comprendre à la population l'important problème social que constitue la violence conjugale. 